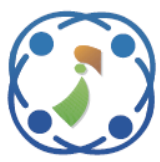

\title{
Multi-Class Arrhythmia Detection using a Hybrid Spatial-Temporal Feature Extraction Method and Stacked Auto Encoder
}

\author{
Vallem Sharmila ${ }^{1 *}$ \\ ${ }^{I}$ Department of Electronics and Communication Engineering, Vignana Bharathi Institute of Technology, \\ Hyderabad, India \\ * Corresponding author's Email: sharmilavallem@gmail.com
}

\begin{abstract}
Cardiovascular disease generally refers to conditions that involve narrowed or blocked blood vessels which can lead to a heart attack, chest pain or stroke. The rate of mortality has been increased among cardiac patients and early detection of the heart disease is important to prevent death. The heart disease can generally be identified at an early stage by analysing irregular heart beat rhythms. The occurrence of abnormal heart beats is known as arrhythmia and the expert system is introduced for detection of arrhythmia. The Electro Cardio Gram (ECG) is considered to be a standard non-invasive diagnosis for Arrhythmia detection and classification of ECG signals was performed for the arrhythmia detection by the researchers in the existing methods. The existing Long Short Term Memory (LSTM)-Auto Encoder (AE) method was failed in selecting prior features to perform feature extraction and also gave rise to overfitting problems. The high level features were need to tackle the issue occurred during data imbalance which gave rise to misclassification of ECG heartbeats. In order to overcome aforementioned issue, the proposed hybrid spatial temporal feature extraction technique extracts salient features and eliminates the exhausted handcrafted features. Initially the ECG signals are acquired from the Massachusetts Institute of Technology-Beth Israel Hospital (MIT-BIH) database. The ECG signals were undergone for the pre-processing stage using butter worth filter. The segmentation of the QRS complex is an important task as the QRS spike in signal determines the abnormality in heart beat using Teager Energy Operator (TEO). The spatial features that provide locations of various natural or artificial boundaries or shapes visualize spatial data of the ECG signals. The temporal features are the time domain features that include Energy of Signal, Zero Crossing Rate, and Maximum Amplitude parameters. These parameters from both spatial and temporal feature forms a hybrid spatial temporal features that are extracted from the QRS complex and are fed to the layers of Deep Neural Network (DNN) based Stacked Auto Encoder (SAE) The hybrid spatial-temporal features determines the type of heart rhythm into 5 prominent classes of Arrhythmia.
\end{abstract}

Keywords: Arrhythmia, Butter worth filter, Deep neural network, Electro cardio gram, Hybrid spatial and temporal features, QRS complex, Stacked auto encoder, Teager energy operator.

\section{Introduction}

In the world, around $31 \%$ of death is occurring due to the cardiovascular disease and $80 \%$ of them are sudden cardiac arrhythmias deaths [1]. Arrhythmia is an abnormal heartbeat rhythm with an irregular frequency occurring in the patients that are determined by using ECG. The symptoms of arrhythmia include chest tightens, low blood pressure, dizziness, sweating, palpitations that can sometimes cause sudden death. Some of the severe types of arrhythmias are rapid atrial fibrillation, persistent ventricular tachycardia, supra ventricular tachycardia, etc. [2]. Many medical imaging techniques are used to diagnose heart problems however, ECG is considered to be a standard non-invasive diagnosis for Arrhythmia. The cost of ECG diagnosis is low and it is very easy to conduct research worldwide [3]. The ECG records the electrical activities of the heart, which is mainly used to diagnose arrhythmia in the clinical practice. The main difficulty faced in diagnosing the arrhythmia is because each and every one is having different rate of heart beats that results with different type of waveforms. To overcome the 
aforementioned problems, different types of heart beats waveforms in ECG signal are analysed which helps in finding arrhythmia in timely manner [4]. The heart beat is composed of $\mathrm{P}$ wave, T wave and QRS complex that represent time domain features to process atria and ventricular depolarization and repolarisation [5]. For each of the cardiac condition, the magnitude of ECG arrhythmia is distinct from where the features are extracted and these features are used for classifying types of arrhythmia [6].In this study, 5 different types of ECG arrhythmia beats are classified as these 5 irregular waveforms are severe and would result as an heart attack, so the present research classifies Arrhythmia into Normal Sinus Rhythm (N), Left Bundle Branch Block (LBBB or L), Right Bundle Branch Block(RBBB or R),Premature Ventricular Contraction (V), and Atrial Premature Beat (A)Rhythm[7].

The many researchers used novel algorithms for detecting arrhythmia disease by means of ECG signals [8]. The time-domain features are extracted in a simple procedure from the segmented ECG signal to detect arrhythmia. However, the extracted features suffered from displacement problems due to selection of $\mathrm{R}$ peak as a fiducial point in an ECG curve. The existing LSTM, ANN, LSTM-AE methods were failed in selecting prior textural features to perform feature extraction which gave rise to over fitting problems [9]. The high level features were needed to tackle the issue occurred during data imbalance which gave rise to misclassification of ECG heartbeats. The proposed hybrid spatial temporal feature extraction technique extracts salient features and eliminates the exhausted handcrafted features. An advantage of the developed model was that the over fitting problem is overcome by the proposed hybrid spatial temporal feature extraction. As the spatial features are extracted within the spatial temporal matrix through full connected layers, SAE cooperates based on DNN capture spatial-temporal features for type of heart rhythm detection that classifies into 5 types of heart beat rhythms accurately. The proposed hybrid spatial temporal feature extraction method initially acquires the ECG signals from the MIT-BIH database [10,11]. The ECG signals are undergone for pre-processing stage in order to eliminate unwanted artifacts in the signals using butter worth filter. The signal is consisting of PQRST wave spike where $\mathrm{P}$ wave describes the depolarization of the right and left atria. The amplitude of this wave is relatively small, because the atrial muscle mass is limited. The QRS complex corresponds to the largest wave, since it represents the depolarization of the right and left ventricles. The $T$ wave depicts the ventricular repolarization.
However, $\mathrm{P}, \mathrm{T}$ waves precise position depends on the heart rate appearing closer to the QRS waves at rapid heart rates so that is the reason we are considering QRS complex as most of QRS peaks are related to heart attacks which is performed by using TEO which is used for detection of the QRS complex by converting spikes into energy envelopes represents the signal determines the QRS peak. The peak QRS is detected and the hybrid spatial temporal features are extracted from the QRS complex. The extracted features were fed to the SAE based on DNN layers and classification of ECG signals into 5 prominent classes such as N, L, R, A and V determines the type of arrhythmia present. The experimental results showed that the proposed hybrid feature extraction showed better accuracy of $99.92 \%$ when compared to existing LSTM-AE.

The organization of the paper is given as follows: Section 2 explains about the contributions of the existing methods and Section 3 describes materials and methods of the proposed technique. Section 4 describes results of the quantitative, comparative analysis of proposed hybrid spatial temporal feature extraction technique. The conclusion and future work of this research is presented in Section 5.

\section{Literature review}

Yao [1] developed a multi class Arrhythmia detection from 12-lead varied length ECG using Attention Based Time Incremental Convolutional Neural Network (ATI-CNN). The traditional methods suffered insufficiency in considering temporal features and thus impacted on CNN during classification as varied length ECG signals. To overcome aforementioned issue, an ATI-CNN was developed where an integration of $\mathrm{CNN}$, attention modules and recurrent cells achieved extraction of both temporal and spatial information from MIT-BIH database. An advantage of the developed model was that, the combination of high quality features was recommended for all types of ECG signals that had varied length, consumed low memory, no overfitting problem in real time system. However, T- wave which is another type of abnormal ECG patterns was not included in the multi class Arrhythmia detection.

Pandey and Janghel [12] developed automatic arrhythmia recognition of ECG signals using different feature methods with LSTM model. The traditional method for arrhythmia classification was time consuming as it has more processing steps. In order to overcome such an issue, LSTM network was presented to detect the heart rhythms form ECG signals accurately. Initially, MIT-BIH arrhythmia database consists of ECG signals was publically 
available to use for data acquisition. The ECG signals were decomposed into features of 45 based on the morphological descriptors, higher order wavelets and $\mathrm{R}-\mathrm{R}$ intervals. Based on the extracted features, the ECG signals classified into 5 arrhythmic rhythms such as N, V, A, L and R rhythms using LSTM. However, separation of ' $A$ ' patterns and normal patterns as well as ' $\mathrm{L}$ ' and ' $\mathrm{V}$ ' patterns were difficult because of similar features present in the patterns were overlapped to each other.

Huang [13] developed an ECG Arrhythmia Classification uses Short-time Fourier transform based Spectrogram and CNN. The Traditional methods performed two steps such as feature extraction and pattern classification in for diagnosing cardiac arrhythmia which was time consuming. In order to overcome such an issue an automatic system in the field of artificial intelligence, such as CNN trained huge amount of data for feature extraction recognised the arrhythmias better than the specialist. The EEG recordings were used from the MIT-BIH arrhythmia database for testing and training the data. The classification of an ECG arrhythmia was performed by using 2 Dimensional Deep Convolutional Neural Network (2D-DCNN) into five types of heart beats such as N, V, A, R and L. The developed model achieved better values of accuracy even without performing pre-processing, noise filtering, feature reduction and extraction manually. However, the numbers of steps were increased for each iteration and the process of convergence faced large fluctuations in each iteration.

Marinho [14] developed a novel ECG feature extraction approach for the classification of cardiac arrhythmia. The existing models failed to select right number neurons hidden layers and to prevent the problem of overfitting due to the complexity of the system. In order to overcome such an issue, a feature extraction process was used to evaluate using Goertzel, Fourier and Higher Order Statics (HOS) and Structural Co-Occurrence Matrix (SCM). The classification was performed by using Support Vector Machine (SVM), Multi-Layer Perceptron (MLP), Bayesian, and Optimum-Path Forest that classified ECG signals into N, R, L, V, and A rhythms and classifiers were compared to each other. An advantage of the developed model showed that the HOS features were achieved best accuracy and reduced the rate of false alarms. The ECG signals classified heartbeats at 106-time rate faster than the traditional approaches. However, an advanced enhancement process was needed in SCM to improve its capacity.

Bouaziz [15] developed an Automatic ECG arrhythmias classification scheme based on the conjoint use of the multi-layer perceptron neural network and a new improved metaheuristic approach. The system performed an automatic classification using MLP neural network and Enhanced Particle Swarm Optimisation (EPSO) algorithm for training. The classification of ECG signals used MIT-BIH database to train classified ECG signals into N, V, A, $\mathrm{L}, \mathrm{R}$ and paced beats. An advantage of the developed model was an improvement in the statistical performance selects the best MLP structure to extract ECG features. However, optimization problems were occurred in the model impacted on decrease in accuracy rate at the time of ECG signal classification.

Chen [16] developed an automated arrhythmia classification based on a combination network of CNN and LSTM. The developed CNN and LSTM approach automatically identified 6 types of ECG signals as Normal, A, V, P, L, R and Sinus Bradycardia rhythms. The developed model used diverse number of subjects that trained the data that improved the network model performance. However, the QRS peak detection was not at all performed in the research which plays an important role in arrhythmia detection that causes computational cost additionally.

Hou [17] Developed LSTM-AE model for ECG Arrhythmias Classification. The LSTM-AE consists of an encoder model that extracted high-level feature information from ECG arrhythmias signals through LSTM network. The decoder model in AE will receive the reconstructed ECG arrhythmia signals from high level features through LSTM network. The classification of ECG signals was obtained as 5 types of heart beat rhythms such as A, V, P, L, R. However, the high level features need to tackle the issue occurred during data imbalance which gave rise to misclassification of ECG heartbeats.

Vedavathi [18] developed Classification for arrhythmia disease based in ECG signals using KNearest Neighbour (KNN) classifier. The developed hybrid features were used for classifying the ECG signals. However, the disease stages needed to be improved using the developed algorithms.

Vedavathi [19] developed an Infinite feature selection based Arrhythmia Classification using Semantic Features and Random Forest. The developed model used semantic features for extraction that was used to utilize and extract the feature vectors from ECG signals. However, the developed model used descriptor level features needed improvement to perform the classification and also more number of arrhythmia heartbeat conditions needed to be analysed further.

Raghu [20] developed feature extraction process that used features like Differential Entropy (DE), 
Peak-Magnitude RMS ratio, Auto Regressive feature that were fed to DNN classifier. However, the classification accuracy was needed still more improvement for performing multi-objective classification method as appropriate features were not utilized.

\section{Proposed method}

The block diagram of the proposed method is shown in Fig. 1.

The block diagram consists of MIT-BIH database, ECG signal processing, ECG signal segmentation,

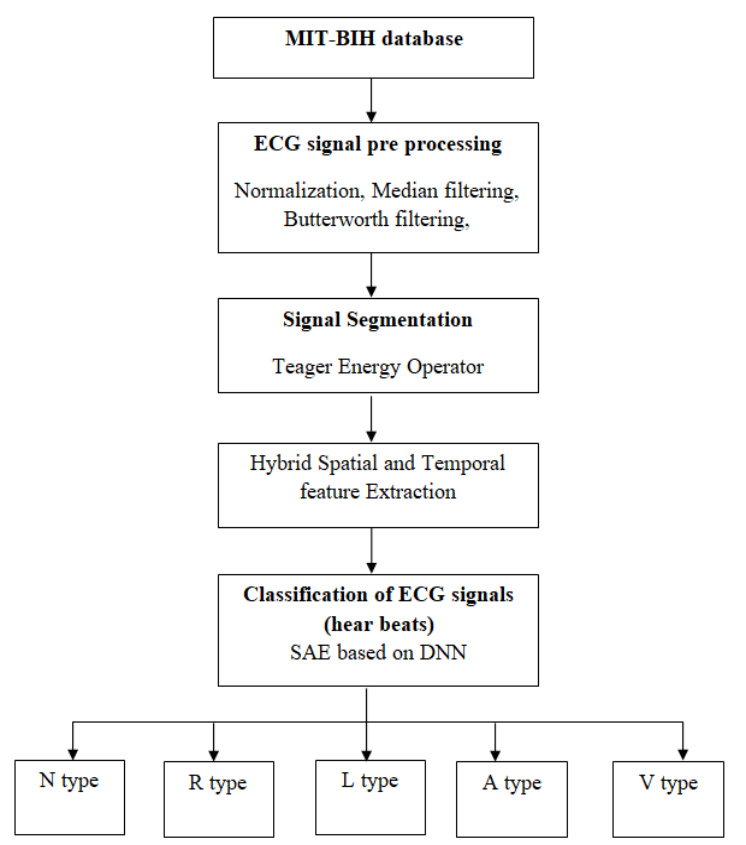

Figure. 1 Block diagram of the proposed hybrid spatial temporal feature extraction model
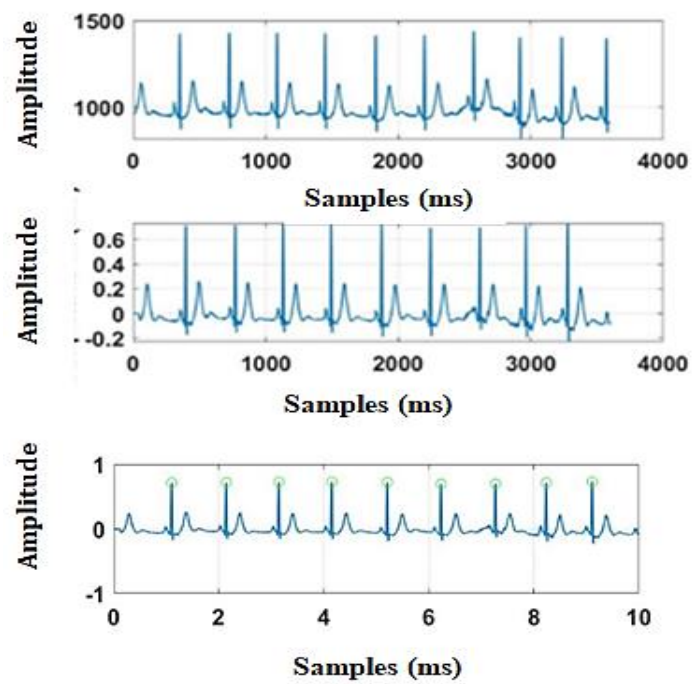

feature extraction from ECG signals and lastly classification of ECG signals.

\subsection{Data collection}

Initially, the MIT-BIH arrhythmia database is used for the research that is available publicly investigates ECG signal classification thereby detects a heart arrhythmia. From 1980, MIT-BIH database is used to perform fundamental research commonly developed a medical device based on the cardiac rhythm and heart diseases [21]. The ECGs of 4000 Holter recordings are included in the MIT-BIH Arrhythmia Database which are obtained in the period of 1975 to 1978 from Beth Israel Hospital Arrhythmia Laboratory. The ECG recordings of $60 \%$ are taken from inpatients that contain 23 records numbered from 100 to 124 . Similarly, a set having 25 records numbered from 200 to 234 are having missing numbers in it. A variety of phenomena that are important clinically are selected from the same set that includes a variety of rare and clinically important stages that represented small sample of Holter recordings. The sample ECG signals of abnormal and normal heartbeats are shown in the Fig. 2.

The abnormal signals of ECG signals are needed to be classified to determine the type of arrhythmia. The ECG signals acquired are undergone for preprocessing technique is explained in the following section.

\subsection{Signal pre-processing}

The ECG records are usually contaminated with different sort of noises, artifacts that are recorded and captured by skin electrodes and the signal usually consisted of high frequency and low frequency noise.
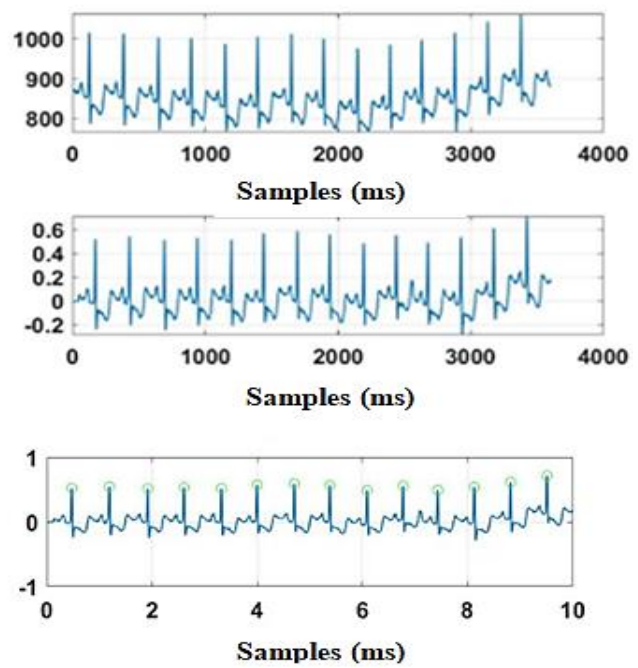

Figure. 2 The signals on the left are the normal heart beats and the signals at the right are the abnormal heart beat ECG signals 


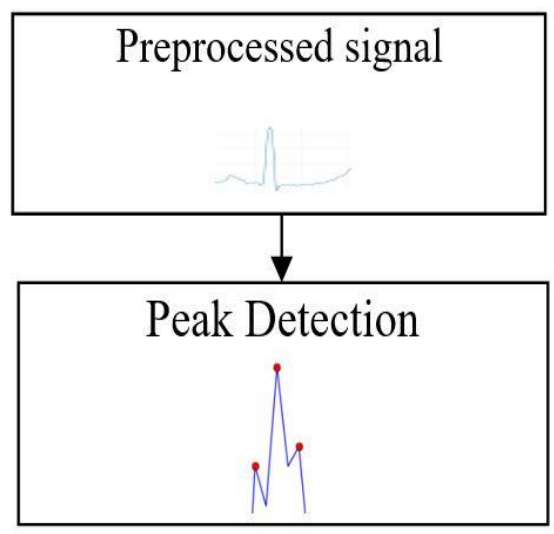

Figure. 3 Pre-processing step

The high frequency noise consisted of Electromyography signal, power line interference etc., and low frequency noise consisted of baseline wander. The pre-processing stage is performed to detect noise accurately so that pre-processed signal samples improve the results. The pre-processing step is performed by using Butter worth filter. As the Baseline wander is a low-frequency noise of around 0.5 to $0.6 \mathrm{~Hz}$ that hinder the detection of peak in the signal. To remove it, a high-pass filter of cut-off frequency 0.5 to $0.6 \mathrm{~Hz}$ which can be performed by using Butter worth high pass filter. The general equation for the butter worth filter is given in Eq. (1).

$$
H(j w)=\frac{1}{\sqrt{1+\varepsilon^{2}\left(\frac{w}{w_{p}}\right)^{2 n}}}
$$

Where,

nis the order of filter

$\Omega$ is equal to $2 \pi f$,

Epsilon $\varepsilon$ is the maximum pass band gain, (Amax).

The pre-processed signals samples obtained using butter worth filter are now undergone for peak detection using TEO which is as shown in the Fig. 3.

\subsection{Peak detection using teager energy operator}

Once the ECG signals are free from noise and artifacts, the pre-processed signal proceeds for peak detection of QRS complex. The QRS complex in the ECG signals plays an important role in tracing the defect present in the heart beats. The QRS complex usually appears visually in the central part of the spike and is considered as a main spike obtained on an ECG line. The spikes are obtained during the depolarization of the left as well as right ventricles and contraction of large ventricular muscles of the heart. Depolarization triggers contraction of the ventricles for the larger tissue mass present in QRS complex than the $\mathrm{P}$ wave. The $\mathrm{P}$ wave describes the depolarization of the right and left atria. The amplitude of this wave is relatively small, because the atrial muscle mass is limited. The QRS complex corresponds to the largest wave, since it represents the depolarization of the right and left ventricles, being the heart chambers with substantial mass. Finally, the T wave depicts the ventricular repolarization. It has smaller amplitude, compared to the QRS complex. However, its precise position depends on the heart rate appearing closer to the QRS waves at rapid heart rates so that is the reason we are considering QRS complex. In order to extract the QRS complex, TEO detects the baseline threshold and thus extracts QRS complex in the ECG signal. The $\mathrm{P}$ and $\mathrm{T}$ waves interfere during QRS complex detection from the ECG signal and this problem is overcome by TEO that minimizes impact of $\mathrm{P}, \mathrm{T}$ waves during QRS complex detection. Once the QRS complex is detected, TEO computation is performed using a moving average technique to determines the envelope of the spikes and smoothen the signal. The following steps are followed for determining QRS complex.

Initially, the sum of kinetic and potential energies is expressed as shown in the Eq. (2) is obtained to calculate the total energy of the signal and is represented in the following Eq. (2).

$$
E=\frac{1}{2} k x^{2}+\frac{1}{2} m x^{2}
$$

Where,

$m$ is the mass of oscillating body

$k$ is the spring constant are used for representing periodic harmonic formula is obtained as shown in Eq. (3).

$$
x(t)=A \cos (\Omega t+\phi)
$$

$\phi$ is the phase shift, $A$ is the position of an oscillating body, $t$ is the time with respect to the variation in the position A.Substituting Eq. (3) in (2) $E$ is obtained as Eq. (4).

$$
E=\frac{1}{2} m A^{2} \alpha A^{2} \Omega^{2}
$$

Eis the total oscillation energy of ECG signal $m$ is the oscillating body's mass

$\alpha$ is the spring constant.

$A$ is the position of an oscillating body

$\Omega$ is the oscillation frequency

An essential signal from harmonic energy generated are calculated by Eq. (4) with the second 
order differential equation as shown in Eq. (5). The following Eq. (6) is a simplified form of TEO for continuous case.

$$
\begin{aligned}
& \psi_{c}[x(t)]=\left[\frac{d}{d t} x(t)\right]^{2}-x(t) \frac{d^{2}}{d t^{2}} x(t) \\
& \psi_{c}[x(t)]=\left[x^{\prime}(t)\right]^{2}-x(t) x^{\prime \prime}(t)
\end{aligned}
$$

Where, $\psi_{c}[x(t)]$ is the energy operator for continuous time $t$

$x(t)$ is the $t^{t h}$ signal component,

$\left[x^{\prime}(t)\right]$ are the first and $\left[x^{\prime \prime}(t)\right]$ second derivatives of $x(t)$

By substituting $n T$ for $t$ in the Eq. (6) give rise to the Eq. (7) as the signals are considered to be discrete. Where, $T$ is the sample period and $n$ is the sample size.

$$
\begin{aligned}
\text { TEO } & =\psi_{d}(x[n]) \\
& =x^{2}[n]-x[n-1] x[n+1]
\end{aligned}
$$

After computing TEO, the signal spikes possess complex energies which are observed in the $\mathrm{P}, \mathrm{T}$ and QRS peaks. The accurate detection of the QRS complex is determined by converting spikes into energy envelopes. The moving average filter is calculated by using the following Eq. (8).

$$
\text { MovingAverage }=\text { filter }\left(h \times j \times \psi_{d}(x[n])\right)
$$

Where, his the rectangle having a length $L$ of the envelope and $j$ is a constant $=1$.

The small amplitude is increased by calculating the square root of moving average using Eq. (9).

$$
S=\sqrt{(\text { MovingAverage })}
$$

Baseline signal's value is decreased below zero, by using the Eq. (10).

$$
\text { Baseline }=\frac{x[n]-\mu}{\sigma}
$$

where $\sigma$ is the standard deviation

$\mu$ is defined as signal average

At last, the peak detection is performed that included following steps as shown in Eq. (11).

$$
\begin{gathered}
\left\{\begin{array}{c}
\operatorname{signal}[n], \text { baseline }<\text { signal }[n], \\
\text { notselected, signal }[n]<\text { baseline },
\end{array}\right\} \\
n=2,3,4, \ldots,
\end{gathered}
$$

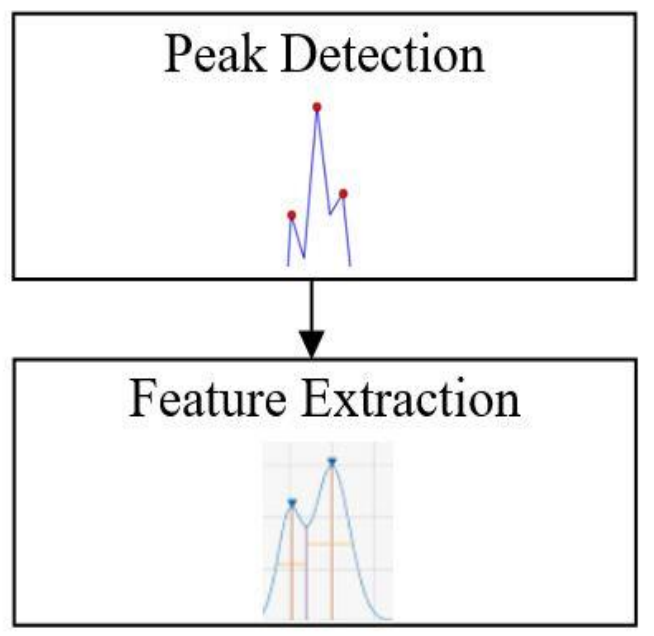

Figure. 4 Peak detection and feature extraction process

Where baseline (0) is the threshold level for peak detection and R peaks are found in an ECG signal by searching the maximum peak of the recognized location of the candidate $\mathrm{R}$ peak in the previous step 10. Once the QRS complex peaks are detected using signal segmentation process, hybrid spatial temporal features are extracted from each of the peak signals as shown in the Fig. 4.

\subsection{Hybrid feature extraction}

The main aim of feature extraction is to transform the original data taken from the dataset that consisted of discriminatory information, thereby reduces the bandwidth of an input data and improves the speed of the process. The proposed hybrid feature extraction process detects conditions of heart disease using Spatial and Temporal domain features. The temporal feature extraction processes on ECG signals based on time domain and the features are extracted from the QRS complex intervals. The spatial features provide locations of various natural or artificial boundaries or shapes to help visualize spatial data of the ECG signals. The spatial feature improves separability of classes. The spatial feature weights in the primal SVM optimization problem and tunes these weights as hyper-parameters. Similarly, the temporal features also known as time domain features, which are simple to extract and that has physical interpretation, such as: the energy of signal, zero crossing rate, maximum amplitude, etc.

The energy of a continuous-time signal $x(t)$ is defined as the area under the squared magnitude of the considered signal given by Eq. (12).

$$
E_{S}=\langle x(t), x(t)\rangle
$$



(13).

The Eq. (11) is further defined as shown in the Eq.

$$
E_{S}=\int_{-\infty}^{\infty}|x(t)|^{2} d t
$$

Where $E_{S}$ is the Energy of the signals $x(t)$ is the continuous amplitude signal

- The amplitude is the distance from the equilibrium position of the medium to a compression or a rarefaction

- The Zero crossing rate (ZCR) is the rate of sign changes along a signal which is expressed as shown in the Eq. (14).

$$
Z C R=\frac{1}{T} \sum_{t=1}^{T}|s(t)-s(t-1)|
$$

Where $s$ is signal of length $T$

The spatial and temporal local features are extracted to form a hybrid features included the mechanism of both spatial and temporal modules. Initially, the spatial domain features assigns weights for feature map that consists of channels contributes to represent ECG signals. Similarly, the temporal domain features assigns weights for temporal segments of a feature map that concentrates more on segments of ECG signals. The two mechanisms are performed one after for each neural block that represented spatial and temporal axes along with local features where spatial features mechanism proceeds and the later temporal mechanism is functioning.

The spatial features that provide locations of various natural or artificial boundaries or shapes visualize spatial data of the ECG signals. The temporal features are the time domain features that include Energy of Signal, ZCR, and Maximum Amplitude parameters. These parameters from both spatial and temporal feature forms a hybrid spatial temporal features that are extracted from the QRS complex and are fed to the layers of Deep Neural Network (DNN) based Stacked Auto Encoder (SAE) in order to classify the abnormal heart beat rhythms.

\subsection{Classification of ECG signals}

The hybrid spatial temporal features are fed to the deep neural network for classification. The SAE is different from ancestral neural networks, which show improvement in approximation properties having ability to represent any non-linear functions. The features are fed into deep learning methods based on the lower level features and it executes a lower level to higher level to form a hierarchy feature level. The spatial features and temporal features obtained during the feature extraction are adjusted and are used by back propagation algorithms. In addition, the SAEs are used in the deep learning model, where SAE acts as DNN and vice versa. The SAE model used to combine with the dropout model in order to train the weight matrix from the signals that are vibrated in the form of spectra. Dropout is an effective strategy that has been proved to reduce over fitting in the training process of neural networks. Auto encoder is an unsupervised learning structure that has three layers such as input layer, hidden layer, and output layer as shown in Fig. 3. An auto encoder training consists of two parts such as encoder and decoder. An encoder is used for mapping the input data into hidden representation, and decoder is referred to reconstructing input data from the hidden representation. The mapped input data from the encoder represents the hidden data and the reconstruction of input data is performed from the hidden representation.

Where $\left\{x_{n}\right\}_{n=1}^{N}$ represents the hidden encoder vector calculated from $x_{n}$ and $\hat{x}_{n}$ is the decoder vector of the output layer. The encoding process is done by using Eq. (15).

$$
h_{n}=f\left(W_{1} x_{n}+b_{1}\right)
$$

Where $f$ is the encoding function, $W_{1}$ is the weight matrix of the encoder, and $b_{1}$ is the bias vector is given by Eq. (16).

$$
\hat{x}_{n}=g\left(W_{2} h_{n}+b_{2}\right)
$$

Where $g$ is the decoding function, $W_{2}$ is the weight matrix of the decoder, and $b_{2}$ is the bias vector. In order to minimize the reconstruction error, the parameter sets of the auto encoder are optimized by using the following Eq. (17).

$$
\phi(\Theta)=\underset{\theta, \theta^{\prime}}{\arg \min } \frac{1}{n} \sum_{i=1}^{n} L\left(x^{i}, \hat{x}^{i}\right)
$$

Where,

$L$ Represents a loss function which is defined as shown in the Equation (18)

$$
L(x, \hat{x})=\|x-\hat{x}\|^{2}
$$

$\arg \min$ is the argument of minimum for the instance which attains smallest value for the loss function.

Stacked Auto Encoders combined with dropout model are applied to train the weight matrix from the frequency spectra of vibration signals. The block 


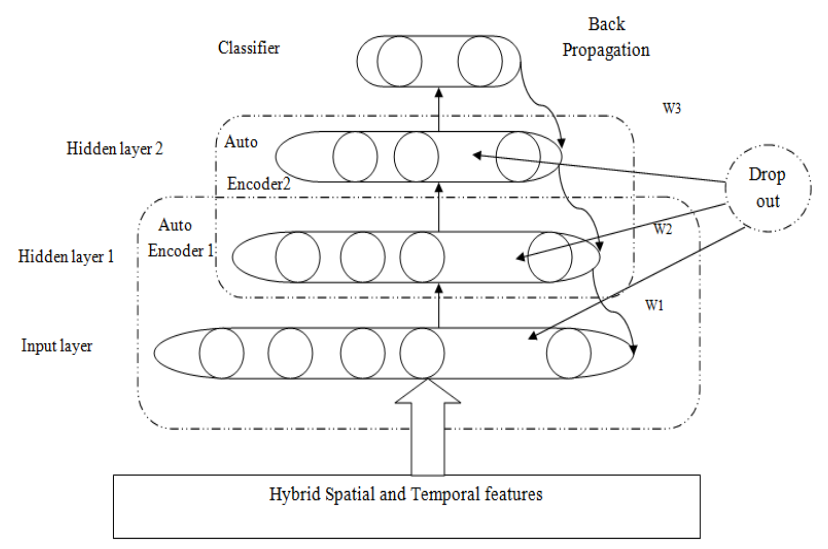

Figure. 5 Block diagram of DNN based SAE

diagram for the DNN based SAE is shown in the Fig. 5.

The DNN are built by using SAE thereby employs the unlabelled data during training set to DNN layer by layer. The DNN based SAE extracts the spatial features within the spatial temporal matrix through full connected layers. The SAE cooperates based on DNN capture spatial-temporal features for type of heart rhythm detection. The classification results obtained from $\mathrm{DNN}$ based $\mathrm{SAE}$ into 5 irregular waveforms which are severe and would result as a heart attack, so the present research classifies Arrhythmia into Normal Sinus Rhythm (N), Left Bundle Branch Block (LBBB or L), Right Bundle Branch Block (RBBB or R), Premature Ventricular Contraction (V), and Atrial Premature Beat (A) Rhythm. The classification results obtained for the proposed hybrid spatial temporal feature extraction is explained in the results section. The SAE based on DNN classifies the MIT-BIH into five different arrhythmia classes such as N, A, V, R and L ECG rhythms as shown in the Fig. 6.

\section{Results}

The experimental setup and performance evaluation of the arrhythmia detection in an ECG signal acquired from the MIT-BIH database is described in this section. The proposed hybrid spatial, temporal feature extraction applies to MIT-BIH database. The MATLAB R2018 software tool and system requirement of 16 GB RAM with i7 processor is used to implement the proposed hybrid spatial temporal feature extraction method. The classification stage used $90 \%$ of dataset for training and $10 \%$ of dataset for testing. The performance measures used to analyse the classified arrhythmia signals results are in terms of Accuracy, F-measure, Sensitivity, Specificity and Precision.

\subsection{Performance analysis}

The proposed hybrid spatial temporal feature extraction method combined spatial and temporal features for ECG arrhythmia classification. The SAE classifier decides the type of heart beat abnormality in ECG signals among, N, A, R, L, or V. In this experimental analysis, the performance of proposed hybrid spatial temporal feature extraction and SAE classifier was compared with existing classifiers such as Long Short Term Memory (LSTM), Artificial Neural Network (ANN), in terms of Accuracy, Specificity, Sensitivity, Precision and F-measure.

\section{i). Accuracy}

Accuracy is defined as the ratio of correctly predicted observations to the total number of observations. The accuracy is calculated by using following Eq. (19).

$$
\text { Accuracy }=\frac{(T P+T N)}{(T P+T N+F P+F N)}
$$

\section{ii). Specificity}

Specificity measures the proportion of correctly identified negatives, which is described by the following Eq. (20).

$$
\text { Specificty }=\frac{T N}{T N+F P} \times 100
$$

\section{iii). Sensitivity}

Specificity measures the proportion of correctly identified positives, which is described by the following Eq. (21).

$$
\text { Sensitivity }=\frac{T P}{T P+F N}
$$

\section{iv). F-measure}

F-measure is the suitable evaluation metrics for finding the effectiveness of abnormality and normality of ECG signals. These measures are statistical variability and a representation of random errors. The equation for F-measure is given in Eq. (22).

$$
F-\text { measure }=\frac{2 T P}{(2 T P+F P+F N)} \times 100
$$

\section{v). Precision}

Precision is also known as Positive Predictive Value which is the ratio of relevant instances to the 

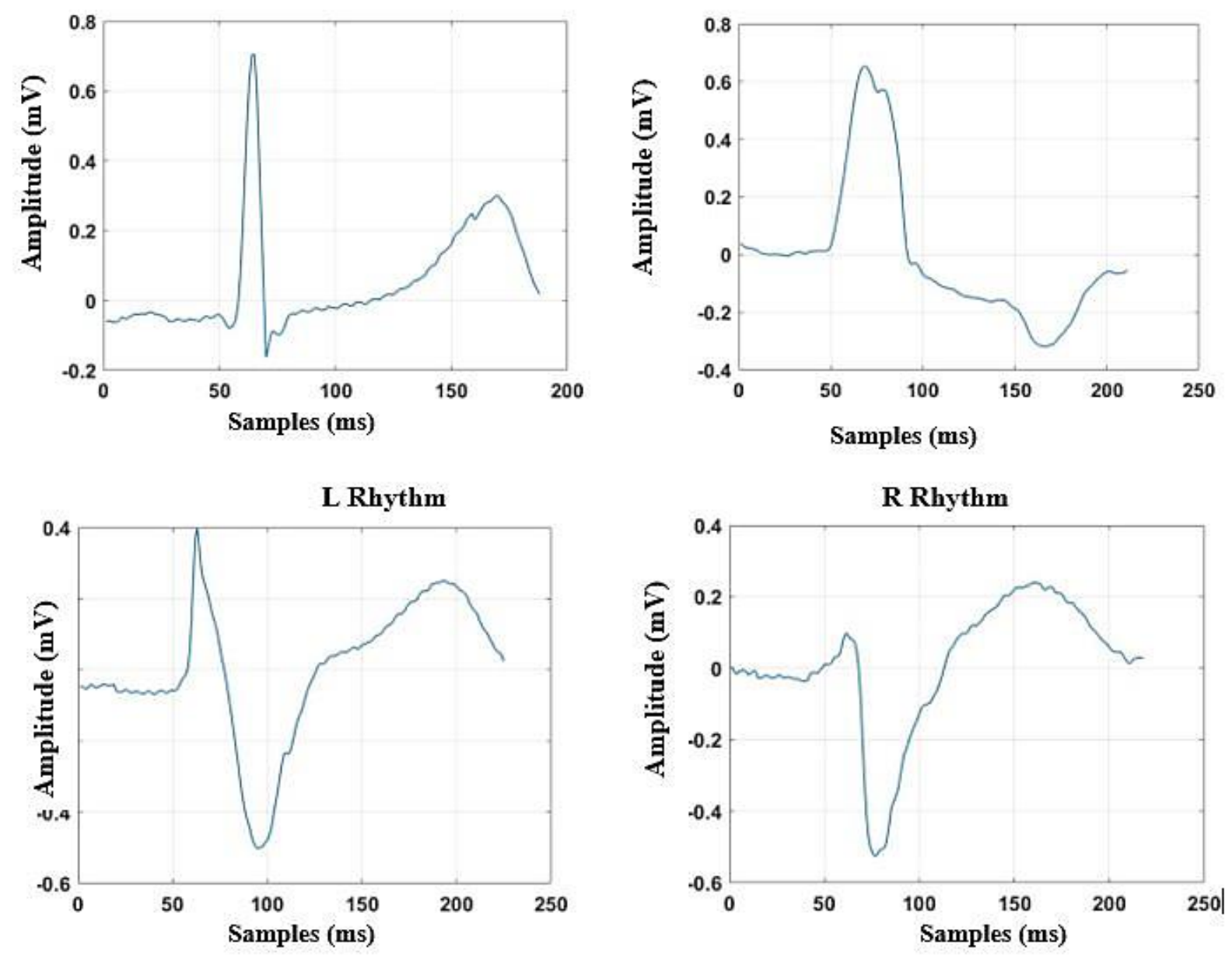

V Rhythm

A Rhythm

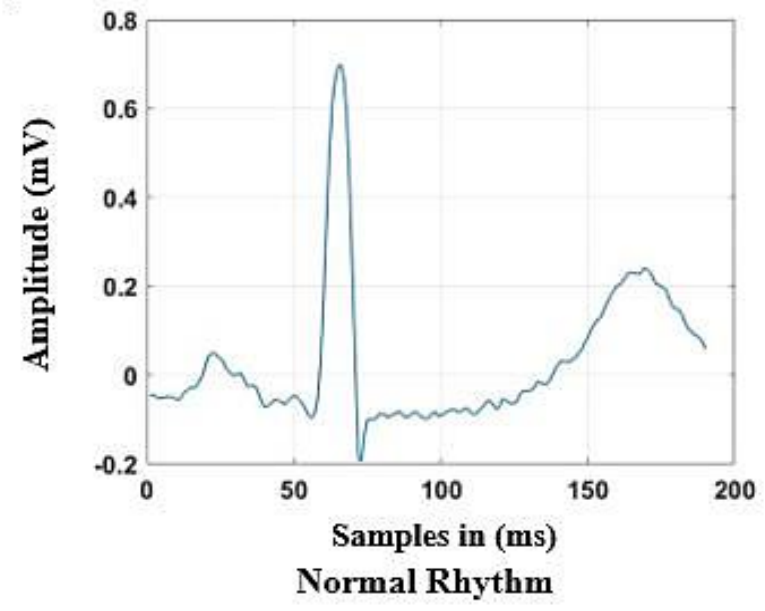

Figure. 6 The five classes of ECG signals

retrieved instances. The equation for Precision is given in Eq. (23).

$$
\text { Precision }=\frac{T P}{T P+F P}
$$

Where, TP, TN, FP, FN are True Positive, True Negative, False Positive and False Negative. The calculation of parameters is described in Table 1.
The obtained results identify arrhythmia and are classified under respective classes. The results show that the $\mathrm{V}$ and $\mathrm{A}$ beats are having high chances of sudden heart attacks compared with N, L, and R beats. The $\mathrm{V}$ and $\mathrm{A}$ beats need to be diagnosed accurately to overcome risk among patients. The results showed that the proposed hybrid spatial temporal feature extraction impact on the DNN-SAE thereby classify 
Table 1. Performance evaluation of the proposed hybrid feature extraction technique

\begin{tabular}{|c|c|c|c|c|c|}
\hline Beat types & Specificity(\%) & Sensitivity(\%) & Accuracy(\%) & Precision(\%) & F1-score (\%) \\
\hline N & 99.41 & 99.37 & 99.16 & 99.84 & 99.17 \\
\hline L & 99.94 & 99.33 & 99.77 & 99.2 & 98.8 \\
\hline R & 99.96 & 99.8 & 99.87 & 99.2 & 99.15 \\
\hline V & 99.94 & 99.82 & 99.92 & 95.7 & 96.13 \\
\hline A & 99.85 & 98.40 & 99.53 & 91.84 & 89.7 \\
\hline Average & 99.82 & 99.344 & 99.65 & 97.156 & 96.59 \\
\hline
\end{tabular}

Table 2. Comparison table for the proposed and the existing methods

\begin{tabular}{|c|c|c|c|c|}
\hline Beat types & Methodologies & Specificity(\%) & Sensitivity(\%) & Accuracy (\%) \\
\hline \multirow[t]{5}{*}{$\mathrm{N}$} & LSTM [12] & 96.45 & 99.31 & 98.60 \\
\hline & ANN [6] & 99.01 & 99.8 & 98.9 \\
\hline & CNN LSTM [16] & 96.33 & 96.28 & 96.30 \\
\hline & LSTM-AE [17] & 99.31 & 99.98 & 87.11 \\
\hline & Proposed & 99.41 & 99.37 & 99.16 \\
\hline \multirow[t]{5}{*}{$\mathrm{L}$} & LSTM [12] & 99.92 & 97.52 & 99.72 \\
\hline & ANN [6] & 99.3 & 98.9 & 99.4 \\
\hline & CNN LSTM [16] & 96.52 & 91.25 & 95.62 \\
\hline & LSTM-AE [17] & 99.88 & 99.21 & 73.27 \\
\hline & Proposed & 99.94 & 99.33 & 99.77 \\
\hline \multirow[t]{5}{*}{$\mathrm{R}$} & LSTM [12] & 99.93 & 98.97 & 99.86 \\
\hline & ANN [6] & 99.9 & 99.6 & 99.8 \\
\hline & CNN LSTM [16] & 99.91 & 98.89 & 99.89 \\
\hline & LSTM-AE [17] & 97.70 & 99.45 & 98.11 \\
\hline & Proposed & 99.96 & 99.8 & 99.87 \\
\hline \multirow[t]{5}{*}{$\mathrm{V}$} & LSTM [12] & 99.63 & 95.18 & 99.32 \\
\hline & ANN [6] & 99.9 & 99.5 & 99.8 \\
\hline & CNN LSTM [16] & 99.83 & 97.92 & 99.79 \\
\hline & LSTM-AE [17] & 99.72 & 86.64 & 99.89 \\
\hline & Proposed & 99.94 & 99.82 & 99.92 \\
\hline \multirow[t]{5}{*}{$\mathrm{A}$} & LSTM [12] & 99.79 & 83.48 & 99.36 \\
\hline & ANN [6] & 99.7 & 97.8 & 99.4 \\
\hline & CNN LSTM [16] & 99.73 & 46.67 & 99.34 \\
\hline & LSTM-AE [17] & 99.96 & 84.72 & 99.57 \\
\hline & Proposed & 99.85 & 98.40 & 99.63 \\
\hline
\end{tabular}

the heart beats into 5 different classes such as $\mathrm{N}, \mathrm{L}$, $\mathrm{A}, \mathrm{V}$, and $\mathrm{R}$. The performance measures for the proposed hybrid spatial temporal feature extraction technique obtained are evaluated in terms of 5 classes $\mathrm{N}, \mathrm{L}, \mathrm{A}, \mathrm{V}$, and $\mathrm{R}$, are summarized in Table 1 . The graphical representation for the Table 1 is shown in the Fig. 5 where the average values for all the 5 arrhythmia classes $\mathrm{N}, \mathrm{L}, \mathrm{R}, \mathrm{A}, \mathrm{V}$ are shown.

\subsection{Comparative analysis}

The ECG arrhythmia classification is performed for the proposed method and is compared with the existing methodologies to analyze the improvement achieved in the proposed hybrid spatial temporal feature extraction technique. The statistical reports are shown in the Table 2 and the graphical representation of overall accuracy obtained for the existing and the proposed hybrid spatial temporal feature extraction model is shown in the Fig. 7.

The comparison results showed that the proposed hybrid spatial temporal feature extraction method achieved better classification accuracy, which shows that the proposed method is convenient to diagnose arrhythmia disease in a better way and ease the work for cardiologist using ECG signal. The comparison of the results for the proposed hybrid spatial temporal feature extraction and with the existing LSTM was examined by Pandey and Janghel, [12] for the arrhythmia detection.

Similarly, Abdalla [6] used ANN for classifying arrhythmia and are compared with the proposed hybrid spatial temporal feature extraction. The extracted hybrid features are fed to SAE classifier and the results obtained is tabulated in the Table 2 . The Table 2 consists of results obtained for the existing methods such as LSTM [12], ANN [6], CNN LSTM 


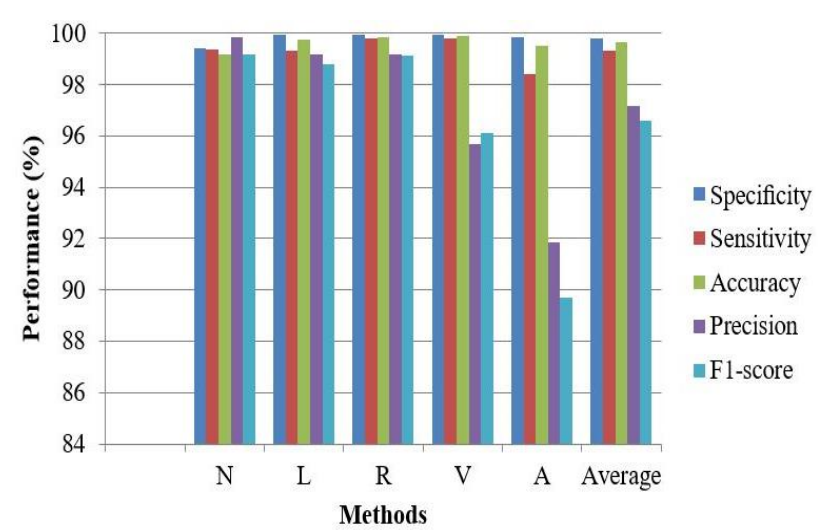

Figure. 7 Graphical representation of the proposed hybrid feature extraction in terms of performance measure

Table 3. Comparison table for the proposed hybrid spatial temporal feature extraction and the existing methods in terms of accuracy

\begin{tabular}{|c|c|c|}
\hline Authors & Classifiers & Accuracy \\
\hline $\begin{array}{l}\text { S.K. Pandey, } \\
\text { and R.R. } \\
\text { Janghel [12] }\end{array}$ & LSTM & 99.354 \\
\hline Abdalla [6] & ANN & 99.46 \\
\hline Chen [16] & CNN LSTM & 98.188 \\
\hline Hou [17] & LSTM-AE & 91.59 \\
\hline $\begin{array}{c}\text { Vedavathi } \\
\text { Gauribidanur } \\
\text { Rangappa [18] }\end{array}$ & $\begin{array}{c}\text { K-Nearest } \\
\text { Neighbour }(\mathrm{KNN})\end{array}$ & $98.40 \%$ \\
\hline $\begin{array}{c}\text { Vedavathi } \\
\text { Gauribidanur } \\
\text { Rangappa [19] }\end{array}$ & Random forest & $94.32 \%$ \\
\hline \multirow[t]{2}{*}{$\begin{array}{c}\text { Raghu } \\
\text { Nanjundegowda } \\
{[20]} \\
\end{array}$} & $\begin{array}{c}\text { Deep Neural } \\
\text { Network (DNN) }\end{array}$ & $98.3 \%$ \\
\hline & Proposed & 99.67 \\
\hline
\end{tabular}

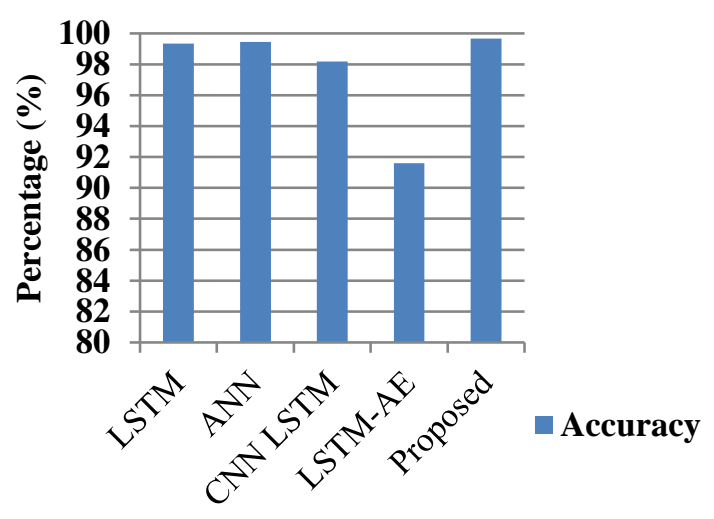

Methodologies

Figure. 8 Comparison graphs for the proposed hybrid spatial temporal feature extraction and the existing methods in terms of accuracy
[16], and LSTM-AE [17] that are used for comparison with the existing methods. The comparison graph for the proposed hybrid spatial temporal feature extraction and the existing methods in terms of accuracy, specificity, sensitivity, precision and F-score which is presented in Fig. 7 and Fig. 8 shows the Comparison graph for the proposed hybrid spatial temporal feature extraction and the existing methods in terms of accuracy as per the values tabulated in Table 3 .

\section{Conclusion}

The Cardiac arrest is the most significant prognostic threat for survival and morbidities worldwide. The existing models have given its huge contribution in determining arrhythmia using ECG signal. However, the existing methods failed in selecting prior features to perform feature extraction which was examined and gave rise to over fitting problems. In order to overcome the issues, present in the existing methods, the proposed hybrid spatial temporal feature extraction technique acquires the ECG signals from the MIT-BIH database and those were undergone pre-processing stage to eliminate unwanted artifacts in the signals using butter worth filter. The pre-processed ECG signals were undergone segmentation in order to extract QRS complex, which is an important spike in signal for determining the abnormality in heartbeat. The peak QRS is detected and the hybrid spatial temporal features are extracted from the QRS complex. The extracted features were fed to the SAE based on DNN layers and the ECG signals classified into 5 prominent classes such as $\mathrm{N}, \mathrm{L}, \mathrm{R}, \mathrm{A}$ and $\mathrm{V}$ that determines the type of present arrhythmia. The classification results were examined in terms of accuracy, precision, sensitivity, specificity and Fmeasure. The proposed hybrid feature extraction technique overcomes the over fitting problems occurred in the existing system thereby achieves the best accuracy of $99.6 \%$. In the future, efficient optimization algorithms can be used to overcome the optimization problems.

\section{Nomenclature: Symbols}

$n$ is the order of filter

$m$ is the mass of oscillating body

$k$ is the spring constant are used for representing periodic harmonic

$A$ is the position of an oscillating body, $t$ is the time with respect to the variation in the position $A$. 
$\psi_{c}[x(t)]$ is the energy operator for continuous time $t$

$x(t)$ is the $t^{t h}$ signal component,

$\left[x^{\prime}(t)\right]$ are the first derivatives

$\left[x^{\prime \prime}(t)\right]$ second derivatives

$T$ is the sample period

$n$ is the sample size.

$h$ is the rectangle having a length $L$ of the envelope

$j$ is a constant

$E_{S}$ is the Energy of the signals

$x(t)$ is the continuous amplitude signal

$s$ is signal of length $T$

$f$ is the encoding function

$W_{1}$ is the weight matrix of the encoder

$b_{1}$ is the bias vector

$x_{n}$ and $\hat{x}_{n}$ is the decoder vector of the output layer.

$f$ is the encoding function

$W_{1}$ is the weight matrix of the encoder

$b_{1}$ is the bias vector

$g$ is the decoding function

$W_{2}$ is the weight matrix of the decoder

$b_{2}$ is the bias vector.

$L$ is a loss function

\section{Greek Symbols}

$\Omega$ is equal to $2 \pi f$,

Epsilon $\varepsilon$ is the maximum pass band gain, (Amax).

$\phi$ is the phase shift

$\mu$ is defined as signal average

$\sigma$ is the standard deviation

\section{Conflicts of Interest}

The authors declare no conflict of interest.

\section{Author Contributions}

The paper conceptualization, methodology, software, validation, formal analysis, investigation, resources, data curation, writing - original draft preparation, writing-review and editing, visualization, supervision and project administration, have been done by $1^{\text {stauthor. }}$

\section{References}

[1] Q. Yao, R. Wang, X. Fan, J. Liu and Y. Li, "Multi-class Arrhythmia detection from 12-lead varied-length ECG using Attention-based TimeIncremental Convolutional Neural Network", Information Fusion, Vol. 53, pp. 174$182,2020$.

[2] C. Chen, Z. Hua, R. Zhang, G. Liu, and W. Wen, "Automated arrhythmia classification based on a combination network of CNN and LSTM",
Biomedical Signal Processing and Control, Vol. 57, pp. 101819, 2020.

[3] F. Murat, O. Yildirim, M. Talo, U. B. Baloglu, Y. Demir, and U. R. Acharya, "Application of deep learning techniques for heartbeats detection using ECG signals-analysis and review", Computers in Biology and Medicine, Vol. 120, pp. 103726, 2020.

[4] H. Wang, H. Shi, K. Lin, C. Qin, L. Zhao, Y. Huang, and C. Liu, "A high-precision arrhythmia classification method based on dual fully connected neural network", Biomedical Signal Processing and Control, Vol. 58, pp. 101874, 2020.

[5] J. Zhang, X. Chen, A. Liu, X. Chen, X. Zhang, and M. Gao, "ECG-based Multi-Class Arrhythmia Detection Using Spatio-Temporal Attention-based Convolutional Recurrent Neural Network", Artificial Intelligence in Medicine, Vol. 106, pp. 101856, 2020.

[6] F. Y. Abdalla, L. Wu, H. Ullah, G. Ren, A. Noor, and Y. Zhao, "ECG arrhythmia classification using artificial intelligence and nonlinear and nonstationary decomposition. Signal", Image and Video Processing, Vol. 13, No. 7, pp. 12831291, 2019.

[7] T. Teijeiro, C. A. García, D. Castro, and P. Félix, "Arrhythmia classification from the abductive interpretation of short single-lead ECG records", In: Proc. of Computing in Cardiology (CinC), pp. 1-4, 2017.

[8] F. Y. Abdalla, L. Wu, H. Ullah, G. Ren, A. Noor, H. Mkindu, and Y. Zhao, "Deep convolutional neural network application to classify the ECG arrhythmia", Vol. 14, pp. 1431-1439, Signal Image and Video Processing, 2020.

[9] J. A. Gutiérrez-Gnecchi, R. Morfin-Magana, D. Lorias-Espinoza, A. del Carmen TellezAnguiano, E. Reyes-Archundia, A. MéndezPatiño, and R. Castañeda-Miranda, "DSP-based arrhythmia classification using wavelet transform and probabilistic neural network", Biomedical Signal Processing and Control, Vol. 32, pp. 4456, 2017.

[10] C. K. Jha and M. H. Kolekar, "Cardiac arrhythmia classification using tunable Qwavelet transform based features and support vector machine classifier", Biomedical Signal Processing and Control, Vol. 59, pp. 101875, 2020.

[11] Y. Xia, H. Zhang, L. Xu, Z. Gao, H. Zhang, H. $\mathrm{Liu}$, and S. Li, "An automatic cardiac arrhythmia classification system with wearable electrocardiogram", IEEE Access, Vol. 6, pp. 16529-16538, 2018. 
[12] S. K. Pandey and R. R. Janghel, "Automatic arrhythmia recognition from electrocardiogram signals using different feature methods with long short-term memory network model", Signal, Image and Video Processing, Vol. 14, pp. 12551263, 2020.

[13] J. Huang, B. Chen, B. Yao, and W. He, "ECG arrhythmia classification using STFT-based spectrogram and convolutional neural network", IEEE Access, Vol. 7, pp. 92871-92880, 2019.

[14] L. B. Marinho, N. de MM Nascimento, J. W. M. Souza, M. V. Gurgel, P. P. Rebouças Filho, and V. H. C. de Albuquerque, "A novel electrocardiogram feature extraction approach for cardiac arrhythmia classification", Future Generation Computer Systems, Vol. 97, pp. 564577, 2019.

[15] F. Bouaziz, H. Oulhadj, D. Boutana, and P. Siarry, "Automatic ECG arrhythmias classification scheme based on the conjoint use of the multilayer perceptron neural network and a new improved metaheuristic approach", IET Signal Processing, Vol. 13, No. 8, pp. 726-735, 2019.

[16] C. Chen, Z. Hua, R. Zhang, G. Liu, and W. Wen, "Automated arrhythmia classification based on a combination network of CNN and LSTM", Biomedical Signal Processing and Control, Vol. 57, pp. 101819, 2020.

[17] B. Hou, J. Yang, P. Wang, and R. Yan, "LSTMBased Auto-Encoder Model for ECG Arrhythmias Classification", IEEE Transactions on Instrumentation and Measurement, Vol. 69, No. 4, pp. 1232-1240, 2019.

[18] V. G. Rangappa, S. V. Prasad, and A. Agarwal "Classification of Cardiac Arrhythmia stages using Hybrid Features Extraction with K-Nearest Neighbour classifier of ECG Signals", International Journal of Intelligent Engineering and Systems, Vol. 11, No. 6, pp. 21-32, 2018.

[19] V. G. Rangappa, S. V. Prasad, and A. Agarwal, "Infinite Feature Selection Based Arrhythmia Classification Using Semantic Features and Random Forest", International Journal of Intelligent Engineering and Systems, Vol. 12, No. 3, pp. 203-213, 2019.

[20] R. Nanjundegowda and V. A. Meshram, "Arrhythmia Detection Based on Hybrid Features of T-wave in Electrocardiogram", International Journal of Intelligent Engineering \& Systems, Vol. 11 No. 1, pp. 153-62, 2018.

[21] G. B. Moody and R. G. Mark, "The impact of the MIT-BIH arrhythmia database", IEEE Engineering in Medicine and Biology Magazine, Vol. 20, No. 3, pp. 45-50, 2001. 